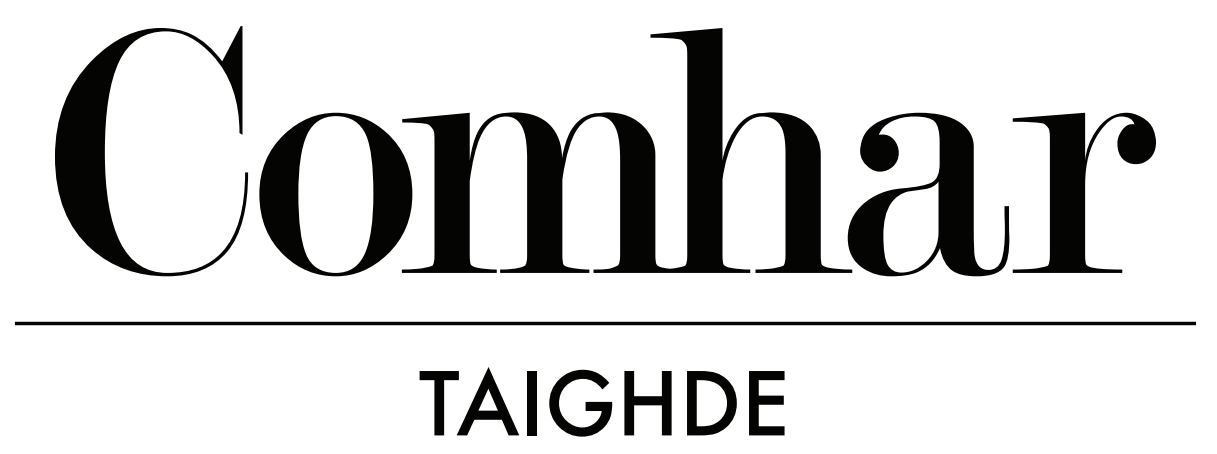

EAGRÁN 7

Nollaig 2021

\begin{abstract}
Alt Taighde
Feasacht teanga an mhúinteora ábhair iarbhunscoile sa tumoideachas: dearcthaí, eispéiris agus tuairimí múinteoirí ábhair i nGaelcholáistí
\end{abstract}

Dáta foilsithe:

1 Nollaig 2021

\section{Cóipcheart:}

(c) Christine Ní Chathasaigh agus T.J. Ó Ceallaigh, 2021

(CC BY-NC)

\section{Seoladh gréasáin:}

https://comhartaighde.ie/eagrain/7/nichathasaighoceallaigh/

\section{Údair:}

Christine Ní Chathasaigh

T.J. Ó Ceallaigh

\section{Comhfhreagras:}

C.NiChathasaigh@mic.ul.ie

TJ.OCeallaigh@mic.ul.ie

Seoladh seasmhach (DOI): https://doi.org/10.18669/ct.2021.03

Is le cabhair deontais i gcomhair tograí Gaeilge a d'íoc an tÚdarás um Ard-Oideachas trí Choláiste na hOllscoile, Corcaigh le tacaíocht Choiste Léann na Gaeilge, Litríocht na Gaeilge agus na gCultúr Ceilteach, Acadamh Ríoga na hÉireann, a fhoilsítear an ríomhiris seo. Aithníonn COMHAR an tacaíocht seo. 


\title{
Feasacht teanga an mhúinteora ábhair iarbhunscoile sa tumoideachas: dearcthaí, eispéiris agus tuairimí múinteoirí ábhair i nGaelcholáistí
}

\author{
Christine Ní Chathasaigh agus T.J. Ó Ceallaigh
}

\section{Achoimre}

Úsáidtear an sprioctheanga mar mheán chun an t-ábhar agus an teanga araon a theagasc sa tumoideachas. Mar sin, is é comhtháthú an ábhair agus na teanga atá lárnach i gcur i bhfeidhm an tumoideachais éifeachtaigh (Cammarata agus Ó Ceallaigh 2020). Dhealródh sé, mar sin, gur cheart go mbeadh ardinniúlacht sa tumtheanga mar bhonn agus mar thaca ag cleachtais teagaisc an mhúinteora ábhair sa tumoideachas iarbhunscoile. Ach, ní mar sin a bhíonn i gcónaí áfach (Nic Réamoinn 2017; Ó Ceallaigh et al. 2019; Ó Treasaigh 2019; Uí Ainiféin 2015). Faoi mar a leathnaíonn an córas tumoideachais in Éirinn agus líon níos mó scoláirí ag freastal ar Ghaelcholáistí (iar-bhunscoileanna ina ndéantar an teagasc trí mheán na Gaeilge taobh amuigh den Ghaeltacht), is suntasaí i bhfad an tábhacht a bhaineann le múinteoirí atá an-inniúil i dteanga an teagaisc agus atá cáilithe chun na réimsí ábhair a theagasc. San alt seo, tugtar tuairisc ar thaighde, ó pheirspictíocht an mhúinteora ábhair sa Ghaelcholáiste, ar na dúshláin mhóra atá le sárú chun déileáil le héilimh an ábhair acadúil agus, ag an am céanna, chun inniúlacht sa tumtheanga a fhorbairt i measc na scoláirí. Bailíodh sonraí ó chúigear múinteoirí ó Ghaelcholáistí éagsúla trí agallaimh agus trí ghrúpa fócais a chur i gcrích. Soláthraítear leis na torthaí léargais ar leith ar na dúshláin shuntasacha a bhíonn ag múinteoirí ábhair sa seomra ranga lán-Ghaeilge iarbhunscoile. Léirítear neamhchinnteacht, easpa tuisceana agus easpa feasachta faoi ról an mhúinteora ábhair ó thaobh na teanga de i dteagasc ábharbhunaithe. Tá tosca áirithe aitheanta a chuireann isteach ar chumas an mhúinteora ábhair cruinnúsáid na Gaeilge a chothú sa rang: inniúlacht teanga neamhleor, easpa oideachais, easpa tacaíochta, easpa treorach. Ag deireadh an ailt, déantar plé ar na himpleachtaí atá ann don fhorbairt ghairmiúil agus don taighde amach anseo. 


\section{Réamhrá}

Cineál ar leith den oideachas dátheangach is ea an tumoideachas lán-Ghaeilge ina ndéantar an t-ábhar a theagasc do scoláirí trí mheán mionteanga atá á foghlaim acu ar scoil. Is do scoláirí nach í an Ghaeilge a gcéad teanga (T1) a chuirtear oideachas dara leibheál lán-Ghaeilge ar fáil de ghnáth i scoileanna lasmuigh den Ghaeltacht ar a dtugtar Gaelcholáistí. I staitisticí ó 2018 / 2019, léirítear go raibh 47 Gaelcholáiste ag freastal ar 3.6\% de na scoláirí scoile go léir i bPoblacht na hÉireann (Gaeloideachas 2019). Is iad an dátheangachas, an délitearthacht agus an ghnóthachtáil acadúil spriocanna an tumoideachais lán-Ghaeilge. In ainneoin an éilimh mhéadaithe ar na cláir sin agus an rath réasúnta a bhíonn orthu, bíonn sé casta go fóill na cláir sin a chur i bhfeidhm agus uaireanta ní éiríonn leo chomh maith is a bhímid ag súil leis (Ó Ceallaigh agus Ní Shéaghdha 2017; Ó Ceallaigh agus Ó Laoire 2021; Ó Duibhir 2018). Tá sé léirithe go gcuireann cláir thumoideachais lán-Ghaeilge dúshláin roimh scoláirí agus roimh mhúinteoirí araon. Ar an gcéad dul síos, cé go mbaineann scoláirí tumoideachais scileanna gabhchumais amach atá ar aon dul le cainteoirí dúchais, léirítear freisin go bhfuil scileanna ginchumais níos ísle acu i dtéarmaí sainiúlacht stór focal, cruinneas gramadaí, agus oiriúnacht úsáide (Ó Duibhir 2018; Walsh 2007). Ina dhiaidh sin, ó tharla go gcuirtear béim mhór ar an ábhar i gcláir thumoideachais, is dúshlán leanúnach é do mhúinteoirí freastal ar an ábhar riachtanach agus ar fhorbairt eolas agus fheasacht na scoláirí ar an teanga (Ní Thuairisg 2014; Ó Ceallaigh et al. 2019; Ó Ceallaigh et al. 2020). Tá taighdeoirí den tuairim gur féidir na dúshláin a bhaineann le forbairt na teanga i measc scoláirí tumoideachais a leagan go páirteach ar easpa feasachta teanga na múinteoirí iarbhunscoile (Ó Ceallaigh et al. 2019). Níl tuiscint iomlán againn go fóill áfach ar conas an cumas is éifeachtaí a dhearadh ar mhaithe le gnóthachtáil an ábhair acadúil agus, go comhuaineach, inniúlacht sa tumtheanga a fhorbairt i measc na scoláirí sa Ghaelcholáiste. San alt seo, dírítear ar pheirspictíochtaí agus ar eispéiris an mhúinteora ábhair sa Ghaelcholáiste ar mhaithe le solas a chaitheamh ar an ábhar seo. Tugtar spléachadh ar dtús san alt seo ar thábhacht na feasachta teanga mar ghné lárnach de chleachtas an mhúinteora ábhair san iar-bhunscoil
lán-Ghaeilge agus féachtar ansin ar dhúshláin áirithe a bhaineann leis an bhfeasacht teanga i gcomhthéacs an tumoideachais in Éirinn. Ansin, tugtar léargas ar réasúnaíocht agus ar mhodheolaíocht an taighde agus cuirtear na torthaí taighde i láthair. Ar deireadh, déantar plé ina leagtar béim ar na buncheisteanna atá ann i gcónaí i dtéarmaí fhorbairt ghairmiúil mhúinteoirí ábhair an tumoideachais agus tugtar achoimre ar threoracha don taighde amach anseo.

\section{Feasacht teanga an mhúinteora ábhair mar thaca agus mar bhonn ag cleachtais teagaisc an tumoideachais iarbhunscoile}

Bíonn foghlaim rathúil ábhair ag brath go mór ar an teanga i réimse an tumoideachais. Bíonn teagmháil fhairsing leis an tumtheanga de dhíth ar fhoghlaimeoirí an tumoideachais (Krashen 1985), mar aon le cleachtadh ar a húsáid (Swain 1985). Téann aisghabháil agus úsáid mhéadaithe i bhfeidhm ar dhoimhneacht na próiseála agus neartaíonn siad na naisc inchinne, rud a fhágann gur fusa teacht orthu seo le linn an ghinchumais (DeKeyser 2007). Faoi mar a thagann méadú ar éilimh chognaíocha an ábhair acadúil, tagann méadú ar na héilimh ó thaobh teanga de chomh maith (Tedick agus Lyster 2020). Mura mbíonn inniúlacht mhaith teanga ag scoláirí sa tumtheanga, ní bheidh ar a gcumas an réimse ábhair a rochtain nó dul i ngleic leis agus iad ag dul ar aghaidh ó bhliain go bliain. Is eol dúinn le fada an lá gurb iad na múinteoirí an acmhainn is tábhachtaí ar leibhéal scoile a théann i bhfeidhm ar ghnóthachtáil na scoláirí. Dá réir sin, tá de chúram gairmiúil ar mhúinteoirí tumoideachais an ghnóthachtáil acadúil a chur chun cinn agus, ag an am céanna, oilteacht agus forbairt na litearthachta sa dara teanga (T2) a fhorbairt. D'fhéadfaí a mhaíomh go gcuireann an fheasacht teanga ar a gcumas do mhúinteoirí ábhair tacú go héifeachtach le foghlaim an ábhair i measc scoláirí i gcomhthéacs chomhtháthú na teanga agus an ábhair sa tumoideachas. Is léir go bhfuil bonn eolais ar leith, tuiscintí doimhne agus inniúlachtaí ríthábhachtacha riachtanach le cur ar chumas múinteoirí an ról casta dinimiciúil sin a chomhlíonadh san oideachas lán-Ghaeilge iarbhunscoile. Sonraítear mar sin go bhfuil leibhéal ard feasachta teanga de dhíth 
ar an múinteoir sa teanga theagaisc chun tabhairt faoin dúshlán sin. Tá trí shainréimse eolais i gceist le feasacht teanga múinteoirí sa tumoideachas: úsáid teanga; eolas faoin teanga; agus saineolas oideolaíoch i dtéarmaí na sprioctheanga sa chomhthéacs ábharbhunaithe (Lyster 2007). Bíonn na réimsí sin ag idirghníomhú lena chéile agus téann siad i bhfeidhm ar a chéile ar bhealaí ar leith. Múnlaíonn an fheasacht teanga acmhainn an mhúinteora tumoideachais chun spriocanna teanga agus gníomhaíochtaí a chruthú ina ndírítear ar riachtanais forbartha na bhfoghlaimeoirí tumoideachais agus ar spriocanna teagaisc an ábhair féin. Tá sé ríshoiléir ón taighde go bhfuil bonn eolais agus tacar sainscileanna oideolaíochta ar leith riachtanach don teagasc sa tumoideachas, lena n-áirítear feasacht teanga an mhúinteora (Cammarata agus Ó Ceallaigh 2020; Lyster 2007; Ó Ceallaigh et al.2018; Tedick agus Lyster 2020). De réir na riachtanas stáit i bhformhór na gcomhthéacsanna idirnáisiúnta, is leor cáilíocht san iar-bhunoideachas ina ndírítear go príomha ar theagasc an ábhair chun dul i mbun teagaisc i suíomh tumoideachais. Is annamh a fhéachtar i gcáilíochtaí den sórt sin ar fhéiniúlachtaí sainiúla an tumoideachais, lena gcuimsítear an t-eolas agus na scileanna atá ag teastáil le haghaidh oideolaíocht an tumoideachais. Dúshlán atá ann i gcónaí is ea ullmhú easnamhach múinteoirí le haghaidh cláir thumoideachais in Éirinn. Cé go bhfuil molta ag taighdeoirí gur cheart sprioc a leagan síos go mbeadh cumas teanga an mhúinteora aitheanta mar chritéir do mhúinteoirí a dteastódh uathu a bheith fostaithe san earnáil agus cáilíocht sa teanga (leibhéal B2 ar a laghad ar Comhchreat Tagartha na hEorpa um Theangacha) mar chritéir cheapacháin le haghaidh múinteoirí sa chóras tumoideachais (Ó Ceallaigh agus Ní Shéaghdha 2017; Ó Ceallaigh et al. 2019), ní bhíonn saincháilíochtaí de dhíth maidir le hinniúlacht teanga de ná cumas an mhúinteora teanga agus ábhar a chomhtháthú in oideolaíocht an oideachais lán-Ghaeilge iarbhunscoile go fóill áfach.

\section{Feasacht teanga an mhúinteora ábhair iarbhunscoile: an lúb ar lár}

In Éirinn, tuairiscítear go bhfuil fíorghanntanas múinteoirí ábhair ann a bhfuil caighdeán sásúil teanga acu le bheith ag obair trí mheán na Gaeilge (Ó Grádaigh
2015). Chomh maith leis sin, léiríonn múinteoirí an ghoirt lán-Ghaeilge an fheasacht ar an bhfreagracht atá orthu a bheith ina samhlacha teanga dá scoláirí ach léiríonn siad easpa muiníne ina gcumais teanga féin (Nic Réamoinn 2017; Ní Thuairisg 2014; Ó Ceallaigh et al. 2020; Ó Treasaigh 2019). Bíonn cruinneas a n-úsáide teanga féin ina ábhar imní agus frustrachais do mhúinteoirí tumoideachais i gcónaí (Nic Réamoinn 2017; Ó Ceallaigh et al. 2020; Uí Ainiféin 2015). Lena chois sin, bíonn drogall ar mhúinteoirí ábhair luathghairme gan aon cháilíocht tríu leibhéal acu sa Ghaeilge a gcaighdeán féin a nochtadh leis na scoláirí ar eagla go gcruthódh sé fadhbanna ó thaobh na bainistíochta ranga de (Ó Ceallaigh et al. 2020). Sonraítear go bhfuil bearnaí suntasacha in eolas faisnéiseach na múinteoirí tumoideachais ar an tumtheanga féin agus ina n-inniúlacht sa teanga sin (Nic Réamoinn 2017; Ní Thuairisg 2014; Ó Ceallaigh et al. 2019; Ó Ceallaigh et al. 2020; Ó Treasaigh 2019; Uí Ainiféin 2015). Aithníonn múinteoirí ábhair an tumoideachais lán-Ghaeilge iad féin mar mhúinteoirí ábhair seachas mar mhúinteoirí teanga agus bíonn deacrachtaí acu an teanga a chomhtháthú leis an ábhar i dteagasc ábharbhunaithe (Ó Ceallaigh et al. 2019; Ó Ceallaigh et al. 2020). Bíonn múinteoirí ábhair ag brath an iomarca ar an ionchur, agus go minic ní mheallann siad go leor foirmeacha teanga ó na scoláirí agus bíonn deacrachtaí acu coincheapa atá níos casta ó thaobh na cognaíochta de lena mbaineann éilimh theanga áirithe a theagasc trí Ghaeilge (Ó Ceallaigh et al. 2019). Músclaíonn na torthaí sin cruacheisteanna suntasacha i ndáil le féiniúlacht múinteoirí tumoideachais. Léiríonn an taighde ar mhúinteoirí a mbíonn ábhar á theagasc acu trí dhara teanga na scoláirí go mbíonn forbairt ghairmiúil nach beag ag teastáil uathu chun an obair a chur i gcrích go héifeachtach. Is gá aird a thabhairt ar an ngné sin agus ní mór féachaint uirthi ar bhonn córasach ar fud leanúntas an oideachais agus na forbartha do mhúinteoirí tumoideachais in Éirinn (Ní Thuairisg 2014; Ó Ceallaigh et al. 2019, 2020; Ó Grádaigh 2015; Ó Duibhir 2018; Walsh 2007). Is cúis bhuartha í an mhoill mhór seo le cur i bhfeidhm na moltaí. Is é sprioc an ailt seo ná léargas a thabhairt ar na creidiúintí, tuairimí agus eispéiris atá ag múinteoirí ábhair i nGaelcholáistí ar an ábhar casta seo. 


\section{Comhthéacs an taighde seo}

Cé go bhfuil fianaise curtha ar fáil go bhfuil ardchaighdeáin, ar an meán, bainte amach ag scoláirí iarbhunscoile tumoideachais in Éirinn (Ó Caollaí 2019), aithnítear freisin riachtanais shainiúla na múinteoirí a bhíonn ag teagasc trí mheán na Gaeilge ag an iar-bhunleibhéal (Ní Thuairisg 2014; Ó Ceallaigh et al. 2019). Tá múinteoirí ábhair ag feidhmiú sa ghort gan mórán tacaíochta, gan mórán oideachais agus go minic gan a bheith líofa ná cumasach i dteanga an teagaisc. Is beag taighde atá déanta ar an ábhar seo, ó pheirspictíocht an mhúinteora ábhair sa tumoideachas iarbhunscoile. Is é sprioc an taighde seo cur lenár dtuiscint ar dhearcthaí, eispéiris agus thuairimí múinteoirí ábhair i nGaelcholáistí. Rud bunriachtanach é an tuiscint sin chun freagairt go hoiriúnach dá sainriachtanais agus, ar an mbealach sin, chun feabhas a chur ar cháilíocht fhoriomlán an teagaisc ábharbhunaithe sa Ghaelcholáiste.

\section{Modheolaíocht}

Dhírigh an taighde cáilíochtúil seo ar an gceist taighde seo a leanas:

\section{Tábla 1: Próifíl na rannpháirtithe}

\begin{tabular}{|l|l|l|l|l|l|}
\hline & Múinteoir 1 & Múinteoir 2 & Múinteoir 3 & Múinteoir 4 & Múinteoir 5 \\
\hline Taithí teagaisc & 2 bhliain & 13 bliain & 13 bliain & Bliain amháin & 7 mbliana \\
\hline $\begin{array}{l}\text { Taithí teagaisc } \\
\text { lán-Ghaeilge }\end{array}$ & 2 bhliain & 13 bliain & 12 bliain & Bliain amháin & 7 mbliana \\
\hline Ábhar & Adhmadóireacht & Ealaín & $\begin{array}{l}\text { Eacnamaíocht } \\
\text { Bhaile }\end{array}$ & $\begin{array}{l}\text { Corpoideachas } \\
\text { \& Gaeilge }\end{array}$ & Corpoideachas \\
\hline Próifíl scoile & $\begin{array}{l}\text { Gaelcholáiste } \\
\text { beag }\end{array}$ & $\begin{array}{l}\text { Gaelcholáiste } \\
\text { cathrach }\end{array}$ & $\begin{array}{l}\text { Gaelcholáiste } \\
\text { beag }\end{array}$ & $\begin{array}{l}\text { Gaelcholáiste } \\
\text { cathrach }\end{array}$ & $\begin{array}{l}\text { Gaelcholáiste } \\
\text { mór }\end{array}$ \\
\hline $\begin{array}{l}\text { Cúlra } \\
\text { scolaíochta }\end{array}$ & $\begin{array}{l}\text { Bunscoil agus } \\
\text { Meánscoil }\end{array}$ & $\begin{array}{l}\text { Bunscoil agus } \\
\text { Meánscoil lán- } \\
\text { Bhéarla }\end{array}$ & $\begin{array}{l}\text { Bunscoil lán- } \\
\text { Bhéarla \& } \\
\text { Gaelcholáiste }\end{array}$ & $\begin{array}{l}\text { Bunscoil lán- } \\
\text { Bhéarla \& } \\
\text { Gaelcholáiste }\end{array}$ & $\begin{array}{l}\text { Gaelscoil agus } \\
\text { Gaelcholáiste }\end{array}$ \\
\hline
\end{tabular}

- Cad iad na dúshláin a bhíonn le sárú ag múinteoirí ábhair sa Ghaelcholáiste chun déileáil le héilimh an ábhair acadúil agus, ag an am céanna, chun inniúlacht sa tumtheanga a fhorbairt i measc na scoláirí?

Ghlac cúigear múinteoirí a bhíonn ag plé le hábhair phraiticiúla éagsúla páirt in agallaimh agus i bhfócasghrúpa idir Feabhra agus Meitheamh 2020. Rinne na rannpháirtithe agallamh leathstruchtúrtha a mhair fiche go tríocha nóiméad a ndearnadh taifeadadh fuaime air. Thug na hagallaimh deis do rannpháirtithe a dtuairimí, a ndearcadh agus a dtaithí maidir leis an ábhar a chur in iúl tríd an bplé. Ghlac na rannpháirtithe páirt i bhfócasghrúpa taifeadta a mhair nócha nóiméad. Bhí ceisteanna an fhócasghrúpa bunaithe ar shonraí a bailíodh in agallaimh roimhe sin. Féach próifíl na rannpháirtithe thíos i dTábla 1 .

Soláthraíodh réasúnaíocht an taighde do na rannpháirtithe leis an tuiscint gurbh fhéidir leo tarraingt siar roimh ré agus tugadh deis dóibh a rannpháirtíocht sa taighde a phlé. Gealladh go gcoinneofaí na sonraí a bailíodh faoi rún agus deimhníodh príobháideacht agus anaithnideacht na rannpháirtithe trí fhaisnéis aitheantais a bhaint de na sonraí.

Rinneadh anailís ar na sonraí cáilíochtúla ar fad de réir mar a bailíodh iad. Leanadh treoracha Seidman 
(1991) a deir gur chóir don taighdeoir marc a chur ar shleachta faoi leith, na sonraí a chur i ngrúpaí agus staidéar a dhéanamh ar na catagóirí chun naisc thuamúla a lorg. Rinneadh tras-scríbhinní de na hagallaimh agus fócasghrúpa. Rinneadh an túschódú a fhad is a bhí tras-scríbhinní á gclóscríobh agus scríobhadh na comhráite díreach mar a tharla siad, gan botúin sa teanga a cheartú. Trí iniúchtaí éagsúla ar an ngaol idir na haonaid sin, cinneadh go raibh cóid áirithe ina bhfo-thacair de chóid eile agus, dá bharr sin, cuireadh le chéile iad (Cohen et al.2006). Ar deireadh, rinneadh na téamaí lárnacha comhaontaithe i ndáil le dearcthaí, eispéiris agus tuairimí múinteoirí ábhair a bheachtú agus a lipéadú. Rinneadh dearcthaí, eispéiris agus tuairimí na múinteoirí ábhair a rangú de réir ceann de na cúig théama seo a leanas:

- Inniúlacht teanga an mhúinteora ábhair

- Teagasc agus foghlaim trí Ghaeilge

- Cruinneas teanga na scoláirí a cheartú

- Dúshláin shainiúla an tseomra ranga ábharbhunaithe i nGaelcholáiste

- Infreastruchtúr tacaíochta.

\section{Torthaí agus plé}

Ní phléifear ach téama amháin san alt seo — inniúlacht teanga an mhúinteora ábhair.

\section{Inniúlacht teanga an mbúinteora}

Tháinig muinín an rannpháirtí as a (h)inniúlacht teanga féin chun cinn go rialta le linn na n-agallamh agus le linn an fhócasghrúpa. Labhair na múinteoirí uilig go hoscailte faoin imní a bhíonn orthu maidir le réim agus le cruinneas na Gaeilge lasmuigh agus laistigh den seomra ranga. Lochtaigh na rannpháirtithe uilig a n-inniúlacht Ghaeilge féin ar an mbonn go bhfuil sí míchruinn, meirgeach agus neamhleor. Is minic le linn agallaimh a d'admhaigh rannpháirtithe go raibh a fhios acu nach raibh siad cruinn, nach raibh a gcuid Gaeilge 'foirfe' agus nach raibh na rialacha gramadaí ar eolas acu. Pléifear an téama seo anois faoi na fo-théamaí seo a leanas: inniúlacht teanga an mhúinteora sa seomra ranga agus inniúlacht teanga an mhúinteora lasmuigh den seomra ranga.

\section{Inniúlacht teanga an mbúinteora sa seomra ranga.}

Léirigh na rannpháirtithe ar fad sa taighde feasacht ghrinn ar an bhfreagracht atá orthu a bheith ina samhlacha teanga dá scoláirí sa rang ábharbhunaithe lán-Ghaeilge. Mar sin féin, léiríodh go leor teannais a bhain leis an bhfreagracht sin. Léirigh siad imní maidir le cruinneas a n-úsáide teanga féin. Agus iad ag cuimhneamh ar na dúshláin a chuir an teagasc ábharbhunaithe lán-Ghaeilge os a gcomhair, shonraigh rannpháirtaithe go bhfuil tionchar suntasach ag inniúlacht teanga an mhúinteora ábhair ar cháilíocht an teagaisc. Thug rannpháirtí amháin an léargas seo a leanas:

Féachann sé nach bhfuil a fhios agam cad atáim ag caint faoi nuair a bhíonn orm smaoineamh faoin nGaeilge (Rannpháirtí, agallamh).

Dar léi, tá an chuma ar an scéal nach bhfuil sí muiníneach faoin méid atá á mhúineadh aici, má tharlaíonn sé go bhfuil sí ag streachailt le teacht ar an struchtúr trí Ghaeilge nó muna bhfuil saintéarmaíocht ar leith aici ar an bpointe agus í i mbun teagaisc. Treocht choiteann a d'eascair as na hagallaimh leathstruchtúrtha agus as an bhfócasghrúpa ab ea imní choiteann i ndáil leis an eolas ar an ngramadach atá ag na rannpháirtithe sa taighde, arb eolas neamhleor go ginearálta é. Bíodh is go léiríonn múinteoirí meas ar ghramadach chruinn ina gcuid oibre, shonraigh siad go mbíonn siad ag mothú imníoch agus cúthaileach, agus nach mbíonn siad lánmhuiníneach maidir lena n-úsáid teanga féin. Léirigh rannpháirtí amháin na leochaileachtaí sin go soiléir:

Níl an cruinneas agam agus uaireanta bíonn faitíos orm mar tá fhios agam go bhfuilim ag déanamh botúin (Rannpháirtí, agallamh).

Ní hamháin gur chuir an t-eolas neamhleor ar an ngramadach isteach ar mhuinín an mhúinteora, ach is léir gur chuir sé isteach ar chumas an mhúinteora ábhair aiseolas ceartaitheach a thabhairt dá scoláirí. Luadh go mbeadh imní ar na múinteoirí ábhair Gaeilge na scoláirí a cheartú i gcás nach mbeadh an leagan cruinn ar a dtoil acu féin: 
Tá an méid sin leagan míchruinn ag dul timpeall, I don't want to add to the problem (Rannpháirtí, agallamh).

Thuairiscigh múinteoir eile go mbíonn a fhios aici uaireanta go bhfuil rud éigin 'mícheart' san abairt, ach nach bhfuil de chumas aici an botún a mhíniú:

ní féidir liom líne nó ciorcal dearg a chur mórthimpeall an bhotúin, mar ní bheidh mé in ann é a mhíniú nuair a chuireann an scoláire ceist orm faoin gcúis (Rannpháirtí, agallamh).

Bhí aontacht láidir ó na rannpháirithe go ndéanann siad neamhaird ar earráidí sa rang nuair nach mbíonn an cumas acu iad a cheartú, nuair a bhíonn siad faoi bhrú ama nó nuair a bhíonn ró-ualach an tsiollabais orthu. Tríd is tríd, shainaithin rannpháirtithe an taighde iad féin mar mhúinteoirí ábhair amháin seachas mar mhúinteoirí teanga i gcomhthéacs theagasc an ábhair. Léiríodh go raibh cinntí maidir le pleanáil agus teagasc déanta ag na rannpháirtithe bunaithe ar an ábhar agus ar an ábhar amháin. Chuir na rannpháirtithe in iúl nach mbíonn scoláirí ag súil go mbeidh cáilíocht na teanga á meas ag múinteoirí ábhair sa chomhthéacs ábharbhunaithe.

Cé gur aithin múinteoirí éilimh theanga na n-ábhar éagsúla, d'admhaigh siad nach raibh na réimeanna teanga uireasacha ábharbhunaithe ar a dtoil acu. Léirigh múinteoirí le taithí teagaisc éagsúil acu i suíomhanna lán-Ghaeilge iarbhunscoile na buarthaí céanna maidir leis na heasnaimh a bhí orthu i dteanga acadúil an ábhair:

Táim ag múineadh trí Ghaeilge le níos mó ná deich mbliana anois agus fós níl na focail go léir agam, cinnte níl na nótaí go léir cruinn, agus nílim cinnte an mbeidh siad riamh (Rannpháirtí, agallamh).

Chomh maith leis sin, is léir go mbíonn ualach oibre sa bhreis i gceist do mhúinteoirí ábhair agus iad ag dul i ngleic le hathruithe curaclaim mar aon leis na héilimh theanga a thagann leo:

Tá an cúrsa chomh nua, táim ag foghlaim faoin gcúrsa fós, níl am agam an téarmaíocht as Gaeilge a fhoghlaim chomh maith. Focail an-randamach agus I suppose nua-aimseartha iad, níl siad agam trí Ghaeilge (Rannpháirtí, agallamh).

Léiríodh freisin go gcasann múinteoirí ábhair ar an mBéarla nuair nach bhfuil inniúlacht chuí teanga acu ailínithe le héilimh chognaíocha an ábhair acadúil:

Uaireanta, bíonn tú i bponc i lár abairte, agus just bíonn sé níos fearr an Béarla a úsáid (Rannpháirtí, fócasghrúpa).

Tá tuiscint agus eolas ar chúrsaí teanga, go háirithe ar an tumtheanga, ríthábhachtach maidir le héifeachtacht na hoibre a dhéanann múinteoir tumoideachais (Tedick agus Lyster 2020; Ó Ceallaigh agus Ó Brolcháin 2020). Is léir ón taighde seo nach mbíonn inniúlacht chuí teanga ag an múinteoir ábhair an t-ionchur agus an t-aiseolas is gá a thabhairt chun inniúlacht na scoláirí sa tumtheanga a fhorbairt. Ní mór don teagasc a chinntiú go n-éiríonn le foghlaimeoirí stór saibhir de nathanna foirmleacha agus inniúlacht riailbhunaithe a fhorbairt (Krashen 1985) mar aon le deiseanna úsáide teanga (Swain 1985) ar mhaithe le teanga a phróiseáil agus a shealbhú (DeKeyser 2007). Mar sin, má úsáidtear an Béarla go minic chun rochtain a fháil ar choincheapa casta ábharbhunaithe, is beag a bheidh sealbhaithe ag scoláirí ó thaobh na Gaeilge de sa seomra ranga ábharbhunaithe.

Thug rannpháirtithe an taighde seo le fios gur chuir easpa muiníne as a gcumas teanga féin, go háirithe a máistreacht ar ghramadach agus ar an stór focal a bhaineann go sonrach leis an ábhar, isteach ar chleachtas teagaisc an ranga. Sonraíonn go leor taighdeoirí go léirítear i dtaithí múinteoirí tumoideachais easnaimh shonracha teanga, easpa muiníne ó thaobh teanga de, agus laigí maidir lena n-eolas faoin teanga (Nic Réamoinn 2017; Ní Thuairisg 2014; Ó Ceallaigh agus Ó Brolcháin 2020; Ó Ceallaigh et al. 2019; Ó Ceallaigh et al. 2020; Ó Treasaigh 2019). Tuigtear chomh maith sa taighde seo gur easpa muiníne i leith a gcumais teanga féin a chuireann srian ar mhéid an aiseolais cheartaithigh, gné atá aitheanta chomh maith ag taighdeoirí eile an ghoirt (Ní Aogáin agus Ó Duibhir 2021; Ó Ceallaigh et al. 2019). Shainaithin rannpháirtithe an taighde seo iad féin mar mhúinteoirí ábhair agus leag siad béim ar leith ar ghnóthachtáil an 
ábhair seachas ar ghnóthachtáil na Gaeilge. Léiríodh i staidéir roimhe seo ar an tumoideachas gurb é teagasc an ábhair an chloch is mó ar a bpaidrín ag múinteoirí ábhair iarbhunscoile an tumoideachais lán-Ghaeilge chomh maith (Ó Ceallaigh et al.2019; Ó Ceallaigh et al. 2020). Maíonn Hoare agus Kong (2008) go dtarlaíonn a leithéid toisc go gcoinnítear múinteoirí tumoideachais agus a gcuid scoláirí cuntasach faoi ghnóthachtáil san ábhar thar rud ar bith eile.

\section{Inniúlacht teanga an mbuinteora lasmuigh den seomra ranga}

Thug na rannpháirtithe ar fad le fios go bhfuil úsáid na Gaeilge i measc na foirne leochaileach mar thopaic. Luadh nach mbíonn daoine ag iarraidh an comhrá a thosú trí Ghaeilge, i gcás nach mbeadh fonn ar an múinteoir eile Gaeilge a labhairt nó i gcás nach mbeadh an mhuinín ag an múinteoir eile Gaeilge a labhairt. Tugadh íomhá shoiléir dúinn nuair a luaigh beirt rannpháirtithe nach mbíonn fonn ar mhúinteoirí Gaeilge a úsáid sa seomra foirne, i gcás go gceapfaidh múinteoirí eile le beagán Gaeilge go bhfuil siad drochbhéasach. Tá blaiseadh de thuairimí na rannpháirtithe ar an ábhar curtha ar fáil i bhFíor 1 thíos:

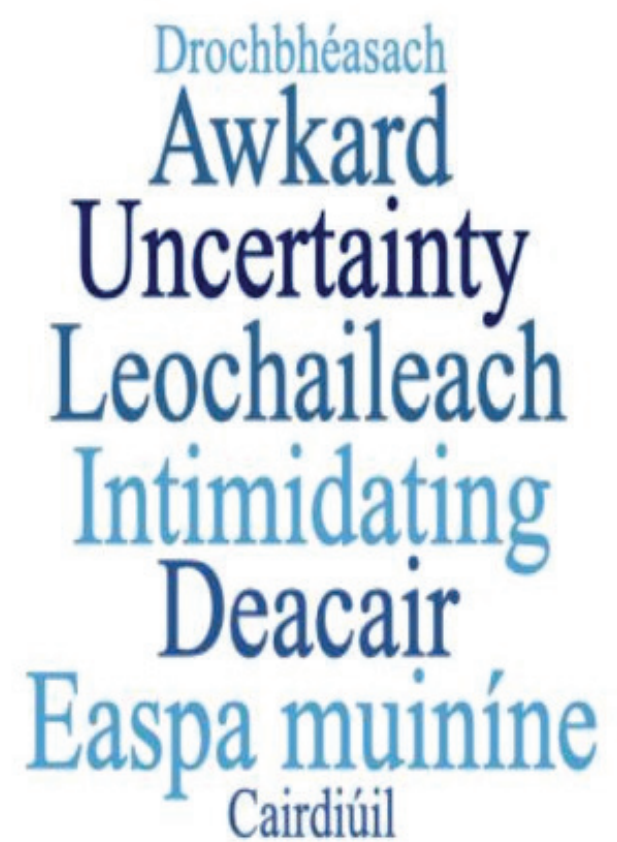

Fíor 1

Focail na rannpháirtithe maidir le húsáid na Gaeilge i measc na foirne
Tagraíodh don réimse leathan cumas teanga a bhíonn ag múinteoirí i nGaelcholáistí chomh maith. Rinne na múinteoirí cur síos freisin ar an tionchar a bhíonn ag múinteoirí atá fostaithe sa scoil, nach bhfuil sásta an Ghaeilge a labhairt nó nach bhfuil de chumas acu Gaeilge a labhairt:

D'fhéadfadh sé a bheith saghas awkward a dhóthain, mar sin in ionad a bheith rude nó an duine a fhágaint amach, labhraímid i mBéarla (Rannpháirtí, fócasghrúpa).

Ábhar buartha atá ar fáil ó mhúinteoir amháin a dúirt go mbíonn imní uirthi ríomhphost a sheoladh chuig foireann na scoile, mar nach bhfuil sí muiníneach aisti féin ag scríobh na Gaeilge. Tá cruinneas a n-úsáide teanga féin ina ábhar imní agus frustrachais do mhúinteoirí tumoideachais léirithe roimhe seo ag taighdeoirí eile chomh maith (Fortune et al. 2008; Ní Thuairisg 2014; Ó Ceallaigh et al. 2020).

Dúradh go mbíonn faitíos ar mhúinteoirí go mbeidh múinteoirí eile ag déanamh measúnaithe ar a gcumas Gaeilge. De réir dealraimh, bíonn céimiúlacht ann ó thaobh na Gaeilge de i scoileanna.

Bíonn sé an-soiléir cé hiad na múinteoirí atá anmhaith ag labhairt agus ag scríobh as Gaeilge, agus níl tú ag iarraidh botúin a dhéanamh ós comhair an duine sin (Rannpháirtí, fócasghrúpa).

Tugadh le fios le linn an taighde gur bagairt mhór í an chéimiúlacht do dhul chun cinn na teanga i measc na foirne agus sa scoil i gcoitinne. Aithníodh níos luaithe go bhfuil ganntanas múinteoirí ann atá inniúil sa Ghaeilge agus cumasach múineadh trí Ghaeilge (Ó Grádaigh 2015), mar sin muna bhfaigheann siad deiseanna cainte chun cleachtadh a dhéanamh ar bhonn rialta ar scoil, ní thiocfaidh feabhas ar an scéal. Tá léargas gonta ar fáil thíos ón taighde a léiríonn go ríshoiléir an tionchar a bhíonn ag na nithe seo ar chleachtas an mhúinteora:

Conas go féidir liom mo Ghaeilge a fheabhsú, mar ní labhraíonn éinne Gaeilge sa seomra foirne, mar sin tá mo Ghaeilge ag éirí níos measa, ní ag éirí níos fearr (Rannpháirtí, agallamh). 
Léiríonn taighde go bhfuil forbairt ghairmiúil shuntasach riachtanach do mhúinteoirí an tumoideachais ionas go mbeidh siad éifeachtach sa chomhthéacs lán-Ghaeilge (Ní Thuairisg 2014; Ó Ceallaigh et al. 2019). Tá luaite ag Ó Ceallaigh agus Ní Shéaghdha go bhfuil gá le 'dian-phlean forbartha gairmiúla atá cuimsitheach agus uileghabhálach, agus atá fréamhaithe i saincheisteanna tábhachtacha agus i sainriachtanais na foirne' (2017: 59). Is fiú go mór mar sin na riachtanais a bhaineann le teanga an tsóisialaithe sa seomra foirne agus teanga na cumarsáide a chur san áireamh i gclár forbartha gairmiúil ar bith don iar-bhunscoil tumoideachais lán-Ghaeilge.

\section{Conclúid}

Gné riachtanach a spreagann cláir thumoideachais rathúla is ea soláthar múinteoirí a bhfuil na hinniúlachtaí éigeantacha teanga acu mar aon leis na cleachtais oideolaíochta ghaolmhara (Ó Ceallaigh agus Ní Shéaghdha 2017). Tugtar le fios sa taighde seo go bhfuil bearnaí suntasacha in eolas faisnéiseach na múinteoirí ábhair ar an tumtheanga féin agus ina n-inniúlacht sa teanga sin agus go bhfuil easpa muiníne acu ina gcumas teanga féin laistigh agus lasmuigh den seomra ranga. Léiríodh easnaimh shonracha teanga, neamhdhiongbháilteachtaí teanga, agus laigí maidir lena gcuid eolais faoin teanga, go háirithe a máistreacht ar an stór focal a bhaineann go sonrach leis an disciplín. Sonraíodh chomh maith go gcuireann na heasnaimh theanga sin bac ar mhúinteoirí ábhair béim a chur ar an teanga i dteagasc ábharbhunaithe. Cé gur fhéach na rannpháirtithe ar an bhfoghlaim leanúnach mar riachtanas gairmiúil chun a n-inniúlachtaí teanga a fhairsingiú, tugadh le fios freisin nach raibh deiseanna úsáide teanga bronnta orthu i gcónaí i gcomhthéacs na scoile chun a bhfeasacht teanga a fhorbairt. D'fhéadfaí torthaí an taighde seo a úsáid le bonn eolais a chur faoi dhearadh agus fhorbairt na forbartha gairmiúla leanúnaí lena bhfreastalaítear ar na hinniúlachtaí teanga éigeantacha agus ar chleachtais oideolaíocha ghaolmhara an mhúinteora ábhair iarbhunscoile. Ar mhaithe le feasacht teanga an mhúinteora ábhair a chur chun cinn, moltar díriú ar na sainghnéithe seo a leanas:
- Deiseanna úsáide teanga a chur ar fáil lasmuigh agus laistigh den Ghaelcholáiste chun máistreacht an mhúinteora ábhair féin ar an tumtheanga a fhorbairt. Le deiseanna úsáide, spreagfar múinteoirí ábhair le machnamh agus le measúnú a dhéanamh ar an bhforás teanga i réimsí spriocdhírithe an ábhair agus tabhairt faoi phleanáil straitéiseach fhéinstiúrtha le cur ar a gcumas feidhmiú go héifeachtach agus go muiníneach mar mhúinteoirí ábhair sa seomra ranga ábharbhunaithe.

- Tacaíocht leanúnach a sholáthar chun tuiscint an mhúinteora ar chórais agus ar struchtúir teanga a chur chun cinn, lena n-áirítear réim agus cruinneas teanga a bhaineann go sonrach leis an ábhar mar aon le teanga bhainistíochta an tseomra ranga ábharbhunaithe sin.

- Freagracht i leith chur i bhfeidhm an chomhtháthaithe ábhar agus teanga a shainaithint mar fhoireann scoile agus pleanáil dá réir. Le polasaí aontaithe le hionchais shoiléire, forbrófar saineolas oideolaíoch agus spreagfar múinteoirí ábhair le hathbhreithniú agus le measúnú criticiúil a dhéanamh ar cháilíocht ghnéithe an tsoláthair ina ranganna féin ar mhaithe le scileanna teanga agus litearthachta a chur chun cinn go leanúnach $\mathrm{i}$ gcomhthéacs an ábhair.

Níos mó ná riamh, tá cultúr an athraithe ag teastáil le go mbainfimid amach an feabhas a éilítear chun caighdeán foghlama na scoláirí a shaibhriú le béim chomhuaineach ar theanga agus ar ábhar sa seomra ranga ábharbhunaithe iarbhunscoile. Tá gá le treoir shainiúil a leagan amach go soiléir do Ghaelcholáistí ar bhonn náisiúnta, a léiríonn na táscairí cáilíochta ó thaobh na teanga de ar mhaithe le freagracht agus ról an mhúinteora ábhair sa phróiseas teagaisc agus foghlama a dhaingniú i gcleachtas na scoile. Moltar freisin go dtabharfar aitheantas do chumas teanga an mhúinteora mar chritéir do mhúinteoirí a dteastódh uathu a bheith fostaithe in earnáil na Gaelscolaíochta agus cáilíocht sa teanga (leibhéal B2 ar a laghad ar Comhchreat Tagartha na hEorpa um Theangacha) mar chritéir cheapacháin do mhúinteoiri sa chóras tumoideachais.

Staidéar mionscála atá sa taighde seo ina gcuirtear i láthair léargas ag am ar leith ar dhearcthaí múinteoirí ábhair i nGaelcholáistí i ndáil leis na dúshláin a 
bhíonn le sárú acu chun déileáil le héilimh an ábhair acadúil agus an teanga ag an am céanna. Is léir go bhfuil deis ann chun leanúint leis an taighde ar na téamaí ar fad a tháinig chun cinn sa staidéar taighde seo. Dá bhrí sin, is gá machnamh eolasach a dhéanamh ar roinnt ceisteanna a bhfuil ceangal acu lena chéile le dul chun cinn a dhéanamh:

- Conas is fearr is féidir feasacht teanga an mhúinteora ábhair a fhorbairt agus a chur chun cinn?

- Conas a théann feasacht teanga an mhúinteora ábhair i bhfeidhm ar an teagasc agus ar an bhfoghlaim sa Ghaelcholáiste?

- Conas a mhúnlaíonn na próisis teagaisc agus foghlama feasacht teanga an mhúinteora ábhair?

\section{GLUAIS}

eolas faisnéiseach: Faisnéis agus eolas maidir le míreanna agus fochórais teanga, amhail sainmhínithe ar fhocail agus rialacha.

eolas faoin teanga: Máistreacht an mhúinteora ar chórais agus ar struchtúir de chuid na teanga, mar aon lena n-eolas ar shainscileanna, léacsacan, gramadach, séimeantaic, pragmataic, ortagrafaíocht, fóineolaíocht, sochtheangeolaíocht, anailís dioscúrsa, teanga na bainistíochta ranga, agus teanga a bhaineann go sonrach le disciplín ar leith. 


\section{SAOTHAIR A CEADAÍODH}

Cammarata, L. agus Tedick, D.J. (2012) 'Balancing Content and Language in Instruction: The Experiences of Immersion Teachers'. In: The Modern Language Journal 96 (2): 251-269. Cammarata, L. agus Ó Ceallaigh, T.J. (eag.) (2020). Teacher Development for Immersion and Contentbased Instruction. Amsterdam: John Benjamins.

Cohen, L., Mannion L. agus Morrison, K. (2007) Research Methods in Education. New York: Routledge.

DeKeyser, R. (2007) 'Skill Acquisition Theory'. In: VanPatten, B. agus Williams, J. (eag.) Theories in Second Language Acquisition: An Introduction. Mahwah: Lawrence Erlbaum Associates: 242-263.

Gaeloideachas (2019) Staitisticí. Ar fáil ag: https://gaeloideachas.ie/is-taighdeoir-me/ staitistici/?lang=ga [faighte 9 Nollaig 2020].

Hoare, P., agus Kong, S. (2008) 'Late Immersion in Hong Kong: Still Stressed or making Progress?'. In: Fortune, T. agus Tedick, D.J. (eag.) Pathways to Multilingualism: Evolving Perspectives on Immersion Education. Clevedon, UK: Multilingual Matters: 242-263.

Krashen, S. (1985) The Input Hypothesis: Issues and Implications. New York: Longman.

Lyster, R. (2007) Learning and Teaching Languages through Content: A Counterbalanced Approach. Amsterdam: John Benjamins.

Ní Aogáin, S. agus Ó Duibhir, P. (2021) 'Iniúchadh ar an Tionchar a bhíonn ag Aiseolas Ceartaitheach ar Chruinneas Gaeilge Daltaí Tumoideachais'. In: Ó Ceallaigh, T. J. agus Ó Laoire, M. (eag.) An Tumoideachas: Deiseanna agus Dea-chleachtais. An Dara Comhdháil Taighde Uile-Oileánda ar an Tumoideachas: Imeachtaí Comhdhála. Baile Átha Claith: An Chomhairle um Oideachas Gaeltachta agus Gaelscolaíochta.

Ní Chathasaigh, C. (2020) Ról agus Freagracht an Mbuinteora Ábhair sa Ghaelcholáiste maidir le Cruinnúsáid na Gaeilge i measc Scoláiri a Chothú: Creidiúintí, Dúshláin agus Cleachtais. Tráchtas M. Oid. san Oideachas Lán-Ghaeilge, Coláiste Mhuire gan Smál, Ollscoil Luimnigh (neamhfhoilsithe).
Ní Thuairisg, L. (2014) Léargas ar Thaithí Mbúinteoiri Iar-bhunscoileanna na Gaeltachta: Dúshláin Ghairmiúla agus Riachtanais Oiliúna. Tráchtas PhD san Oideachas, Coláiste Phádraig, Droim Conrach (neamhfhoilsithe).

Nic Réamoinn, E. (2017) Forbairt Teanga an Mhuiinteora sa Ghaelcholáiste. Tráchtas M. Oid. san Oideachas Lán-Ghaeilge, Coláiste Mhuire gan Smál, Ollscoil Luimnigh (neamhfhoilsithe).

Ó Caollaí, E. (2019) 'Feeder Schools: Irish Medium Education a Feature of Top Progression Tables'. In: Irish Times, 3 Nollaig 2020. Ar fáil ag: https:// www.irishtimes.com/news/education/feederschools/feeder-schools-irish-medium-educationa-feature-of-top-progression-tables-1.4099580 [faighte 10 Nollaig 2020].

Ó Ceallaigh, T.J. agus Ó Laoire, M. (eag.) (2021) An Tumoideachas: Deiseanna agus Dea-chleachtais. An Dara Combdháil Taighde Uile-Oileánda ar an Tumoideachas: Imeachtaí Combdhála. Baile Átha Claith: An Chomhairle um Oideachas Gaeltachta agus Gaelscolaíochta.

Ó Ceallaigh, T.J. (2020) 'Feasacht Teanga Múinteoirí a nochtadh tríd an Staidéar Ceachta'. In: Taighde agus Teagasc 7: 171-186.

Ó Ceallaigh, T.J. agus Ní Shéaghdha, A. (2017) Criteirir Aitheantais Cui um dhearbhú Cáiliochta agus Dea-chleachtais do Bhunscoileanna agus Iarbhunscoileanna Lán-Ghaeilge ar bhonn uile Éireann. Baile Átha Cliath: GAELSCOILEANNA.

Ó Ceallaigh, T.J. agus Ó Brolcháin, C. (2020) 'Trasteangú san Oideachas Lán-Ghaeilge: Deiseanna agus Dúshláin'. In: Taighde agus Teagasc 7: 155-170.

Ó Ceallaigh, T.J., Dennehy, N., Ní Chonaill, M. agus O’Connor, A. (2020). 'Tús Maith? Iniúchadh ar Dhearcthaí Múinteoirí faoi Oiliúint agus Múinteoirí ag tosú amach sa Ghairm maidir leis an Teagasc in Iar-bhunscoileanna Lán-Ghaeilge, agus ar a dTaithí ar an Teagasc sin'. Féilte 2020, Teaching Council Festival of Education in Learning and Teaching Excellence, 3 Deireadh Fómhair 2020 (seimineár gréasáin).

Ó Ceallaigh, T.J., Hourigan, M. agus Leavy, A. (2018) 'Developing Potentiality: Pre-service Elementary Teachers as Learners of Language 
Immersion Teaching'. In: International Journal of Bilingual Education and Bilingualism. Ar fáil ag: https://www.tandfonline.com/doi/full/10.1080/13 670050.2018.1489779 [1 Feabhra 2021].

Ó Ceallaigh, T.J., Leavy, A. agus Hourigan, M. (2019) 'Comhtháthú an ábhair agus na teanga i gcomhthéacs an tumoideachais: Múinteoirí faoi Oiliúint i mbun Éabhlóide tríd an Staidéar Ceachta'. In: COMHARTaighde 5. Ar fáil ag: https://comhartaighde.ie/eagrain/5/oceallaighhourigan-leavy/ [faighte 1 Feabhra 2021].

Ó Ceallaigh, T.J., Ó Laoire, M. agus Uí Chonghaile, M. (2019) Combtháthú ábhar agus teanga san iarbhunscoil lán-Ghaeilge/Ghaeltachta: I dtreo eispéiris forbartha gairmiúla chun dea-chleachtais a nochtadh. Baile Átha Cliath: An Chomhairle um Oideachas Gaeltachta agus Gaelscolaíochta. Ó Duibhir, P. (2018) Immersion Education: Lessons from a Minority Language Context. Bristol: Multilingual Matters.

Ó Grádaigh, S. (2015) Soláthar Múinteoirí Ábhar do na bIarbhunscoileanna Lán-Ghaeilge agus Gaeltachta. Baile Átha Cliath: An Chomhairle um Oideachas Gaeltachta agus Gaelscolaíochta.

Ó Treasaigh, D. (2019) Conas mar a Théann an TFC i bhfeidhm ar Inniúlacht an Mhúinteora ag an Dara Leibhéal. Tráchtas M. Oid. san Oideachas LánGhaeilge, Coláiste Mhuire gan Smál, Ollscoil Luimnigh (neamhfhoilsithe).

Seidman, I.E. (1991) Interviewing as Qualitative Research: A Guide for Researchers in Education and the Social Sciences. New York: Teachers College, Columbia University.

Swain, M. (1985) 'Communicative Competence: Some Roles of Comprehensible Input and Comprehensible Output in its Development'. In: Gass, S. agus Madden, C. (eag.) Input in Second Language Acquisition. New York: Newbury House: 235-256.

Tedick, D.J. agus Lyster, R. (2020) Scaffolding Language Development in Immersion and Dual Language Classrooms. New York: Routledge. Uí Ainiféin, M. (2015) An Fhogblaim Chombtháite Ábhar agus Teanga: Meon agus Mianach.

Tráchtas M. Oid. san Oideachas Lán-Ghaeilge, Coláiste Mhuire gan Smál, Ollscoil Luimnigh (neamhfhoilsithe).
Walsh, C. (2007) Cruinneas na Gaeilge Scriofa sna bIar-Bhunscoileanna Lán-Ghaeilge i mBaile Atha Cliath. Baile Átha Cliath: An Chomhairle um Oideachas Gaeltachta agus Gaelscolaíochta. 